International Journal of Modern Physics D

\title{
A GENERALIZATION OF UNIMODULAR GRAVITY WITH VACUUM-MATTER ENERGY EXCHANGE
}

\author{
F.R. KLINKHAMER \\ Institute for Theoretical Physics, Karlsruhe Institute of Technology (KIT), \\ 76128 Karlsruhe, Germany \\ frans.klinkhamer@kit.edu
}

\begin{abstract}
An effective theory of gravity in the infrared is proposed, which involves the determinant of the metric relative to the determinant of a prior metric taken to be that of Minkowski spacetime. This effective theory can be interpreted as a generalization of unimodular gravity. In a cosmological context with ultrarelativistic or cold matter, the resulting field equations have only one solution, empty Minkowski spacetime (selected by the prior metric of the theory). The introduction of energy exchange between vacuum and matter gives rise to nonstatic cosmic solutions. It is found that Minkowski spacetime (from the prior metric) appears as an attractor of the dynamic equations. A further result is that energy-momentum conservation of any localized material system is violated in a nonconstant gravitational background. The impact for experiment appears, however, negligible if the vacuum-energy mass scale is of order meV.
\end{abstract}

Journal: Int. J. Mod. Phys. D 26 (2017) 1750006

Preprint: arXiv:1604.03065

Keywords: General relativity; dark energy; cosmology; cosmological constant.

\section{Introduction}

A novel point of view on the long-standing cosmological constant problem is provided by so-called unimodular gravity $\frac{1|2| 3|4| 5 \mid}{}$ In this approach, the cosmological constant $\Lambda$ does not appear as input of the gravitational field equation but arises as a constant of integration. However, unimodular gravity does not provide the value of $\Lambda$.

Here, we present a theory which interpolates between standard general relativity and unimodular gravity. The difference with the standard formulation of unimodular gravity is that a value for $\Lambda$ is built in, namely, $\Lambda=0$. Specifically, we find that the solution of the gravitational field equation in a cosmological context approaches a metric with determinant minus 1 and has a vanishing cosmological constant (vacuum energy density).

Still, the new theory does not have the main advantage of the unimodulargravity approach, the automatic cancellation of zero-point energies. These zeropoint energies and other contributions to the vacuum energy density are to be 
cancelled dynamically by microscopic degrees of freedom $[6$ and this cancellation can be described macroscopically by the so-called $q$-theory $[7|8| 9|10| 11$ The present article assumes that $q$-theory (or any other viable compensation mechanism) provides for a cancellation of the zero-point energies and focusses on the gravitation theory emerging in the infrared.

Our attitude as to which type of gravitation theory emerges is entirely agnostic and, in principle, we wish to explore all possible terms involving the metric field (the gravitational field definitely known to exist). For a theory valid over large distances, we then look for terms with the lowest number of derivatives of the metric. It turns out that, apart from the term corresponding to the cosmological constant $\Lambda$, there is another term without derivatives. This term involves the determinant of the metric, hence the connection to unimodular gravity. But there is a price to pay if we wish to maintain general covariance, as will be explained below.

\section{Generalized unimodular gravity}

\subsection{Setup}

Consider a modest extension of standard general relativity with the following action:

$$
\begin{aligned}
S & =S^{\text {grav }}+S^{M}=-\int_{\mathbb{R}^{4}} d^{4} x \sqrt{-g}\left(\frac{R}{16 \pi G_{N}}+\epsilon(\sigma)+\mathcal{L}^{M}[\psi]\right), \\
g & \equiv \operatorname{det} g_{\alpha \beta} \\
\sigma & \equiv \sqrt{-g} / \sqrt{-g_{0}}
\end{aligned}
$$

where the metric $g_{\alpha \beta}$ has a Lorentzian signature $(-,+,+,+)$ and where $g_{0}=g_{\text {Mink }}$ corresponds to the determinant of the Minkowski metric $g_{\alpha \beta}^{\mathrm{Mink}}$ with the same signature. Remark that $\sigma$ is a scalar, as it is the ratio of two scalar densities of equal weight. The theory (10) is, in fact, invariant under general coordinate transformations with arbitrary nonzero values of $\operatorname{det}\left(\partial x^{\prime \alpha} / \partial x^{\beta}\right)$. The price to pay for having unrestricted general coordinate invariance is the introduction of a prior metric giving the scalar density $g_{0}$. As we will see in the next subsection, this price is relatively small, just a "parameter" of a potential term in the action.

The field $\psi$ in the Lagrange density $\mathcal{L}^{M}$ of the matter action $S^{M}$ stands for a generic matter field containing, for example, all the fields of the standard model of elementary particle physics. Strictly speaking, $\mathcal{L}^{M}$ also depends on the metric or the vierbeins via the covariant derivatives, but we keep this dependence implicit. In principle, it is also possible to make the gravitational coupling $G$ and the parameters of $\mathcal{L}^{M}$ dependent on $\sigma$, that is, to have $G=G(\sigma)$ replacing Newton's constant $G_{N}$ and $\mathcal{L}^{M}=\mathcal{L}^{M}(\sigma, \psi)$. But, here, we consider the simplest possible theory with action (1a) and the scalar $\sigma$ appearing only in the potential term $\epsilon(\sigma)$.

The Einstein gravitational field equation from (1a) takes the standard form,

$$
R^{\alpha \beta}-\frac{1}{2} g^{\alpha \beta} R=-8 \pi G_{N}\left(T_{V}^{\alpha \beta}+T_{M}^{\alpha \beta}\right),
$$


with the standard energy-momentum tensor of the matter fields,

$$
T_{M}^{\alpha \beta} \equiv \frac{2}{\sqrt{-g}} \frac{\delta S_{M}}{\delta g_{\alpha \beta}}
$$

and the vacuum-energy term

$$
\begin{gathered}
T_{V}^{\alpha \beta}=\rho_{V}(\sigma) g^{\alpha \beta}, \\
\rho_{V}(\sigma)=\epsilon(\sigma)+\sigma \frac{d \epsilon(\sigma)}{d \sigma} .
\end{gathered}
$$

The crucial observation here is that the vacuum energy density $\rho_{V}$ of the gravitational field equation differs from the vacuum energy density $\epsilon$ of the action. A similar difference has been found before in the context of condensed matter physics 6 and certain relativistic theories! $[7 / 89$

For concreteness, take the following quadratic Ansatz for the function $\epsilon(\sigma)$, with corresponding $\rho_{V}(\sigma)$ from (4b):

$$
\begin{aligned}
\epsilon(\sigma) & =m^{4}\left[\frac{1}{3}\left(\sigma-\frac{3}{2}\right)^{2}+\frac{1}{4}\right], \\
\rho_{V}(\sigma) & =m^{4}[1-\sigma]^{2},
\end{aligned}
$$

where $m$ is a new mass scale. Admittedly, we have fine-tuned (5a), in order to arrive at (5b). See Sec. 4.2 for discussion on how the underlying physics, perhaps analogous to known condensed-matter-physics systems, could produce (5b) close to equilibrium.

The theory given by Eqs. (11) and (5) can be considered as an interpolation between standard general relativity and unimodular gravity. The limit $m \rightarrow 0$ reproduces standard general relativity and the limit $m \rightarrow \infty$ gives unimodular gravity, in the sense that $\operatorname{det} g_{\alpha \beta}(x)$ for standard Cartesian coordinates is fixed dynamically to a constant value. As an effective theory, we may consider setting $m \sim \mathrm{meV}$, in line with astronomical observations of the present accelerating universe 12

In this article, we consider only a prior metric corresponding to Minkowski spacetime, but the afore-mentioned astronomical observations suggest the relevance of a prior metric corresponding to de-Sitter spacetime, which we briefly discuss in Appendix A.

\subsection{Equilibrium conditions and linearized gravity}

The Ansatz (5) implements the following equilibrium conditions at $\sigma_{0}=1$ :

$$
\begin{aligned}
& \rho_{V}\left(\sigma_{0}\right)=0, \\
& {\left[\frac{d \rho_{V}(\sigma)}{d \sigma}\right]_{\sigma_{0}} }=0, \\
& {\left[\frac{d^{2} \rho_{V}(\sigma)}{d \sigma^{2}}\right]_{\sigma_{0}}>0 . }
\end{aligned}
$$


In this way, we have a generalization of unimodular gravity, given by Eqs. (1) and (5), which reproduces the linearized theory of general relativity (here, in the harmonic gauge) but differs in higher order:

$$
\begin{aligned}
g_{\alpha \beta} & =\eta_{\alpha \beta}+h_{\alpha \beta}, \\
\partial_{\alpha} h_{\beta}^{\alpha} & =\frac{1}{2} \partial_{\beta} h_{\alpha}^{\alpha}, \\
\square h_{\alpha \beta} & =-16 \pi G_{N}\left[\widehat{T}_{\alpha \beta}^{M}-\frac{1}{2} \eta_{\alpha \beta} \widehat{T}_{\gamma}^{M \gamma}\right]+\cdots,
\end{aligned}
$$

with the Minkowski metric $\eta_{\alpha \beta} \equiv[\operatorname{diag}(-1,1,1,1)]_{\alpha \beta}$ for standard Cartesian coordinates, the flat-spacetime d'Alembertian $\square \equiv \partial_{0}^{2}-\nabla^{2}$, and the flat-spacetime energy-momentum tensor $\widehat{T}_{\alpha \beta}^{M}$ containing only $\eta_{\alpha \beta}$.

The equilibrium conditions (6) make that the contribution (4a) to the gravitational field equation is second-order in $\left(\sigma_{0}-\sigma\right)$ and corresponds to quartic order in $h$ after gauge fixing (see below). Strictly speaking, the condition (6a) suffices to recover the linearized theory of general relativity, but we add the equilibrium condition (6b) and the further stability condition (6c). These conditions arise naturally in the framework of $q$-theory 79111 : conditions (6a) and (6b) come from the selfadjustment of the conserved vacuum variable $q$ [with chemical potential $\mu=d \epsilon / d q$ and $\left.\rho_{V}(q)=\epsilon(q)-\mu q\right]$ by use of the Gibbs-Duhem relation for an isolated selfsustained system without external pressure, while condition (6c) corresponds to having a positive isothermal compressibility. See Sec. 4.2 for further discussion.

For gravitational waves in the transverse-traceless (TT) gauge (see, e.g., Sec. 35.4 of Ref. 13), we obtain from (7c) without source terms the standard linear wave equation:

$$
\square h_{\alpha \beta}^{T T}=0,
$$

which gives standard propagation,

$$
k^{2}=\eta^{\alpha \beta} k_{\alpha} k_{\beta}=0 .
$$

The standard propagation behavior (9) differs from the generic propagation behavior of, for example, Rosen's bi-metric theory discussed in Ref. 14. The crux is that, for us, the prior metric $\eta_{\alpha \beta}$ enters only in the potential term $\epsilon$ of (1a) and not in the kinetic terms $R$ and $\mathcal{L}^{M}$. In this way, the d'Alembertian for gravitational waves is the same as the one for electromagnetic waves from the Maxwell term $F_{\alpha \beta} F^{\alpha \beta}$ contained in $\mathcal{L}^{M}$.

In closing, we display the scalar $\sigma$ in terms of the physical degrees of freedom. Consider a plane gravitational wave in the TT gauge propagating in the 3 -direction and denote the two polarizations by $h_{+}$and $h_{\times}$. The $2 \times 2$ sub-matrix for $h_{\alpha \beta}$ has $\pm h_{+}$on the diagonal positions and $h_{\times}$on the off-diagonal positions. We then have the determinant

$$
\operatorname{det} g_{\alpha \beta}=-\left(1-h_{+}^{2}-h_{\times}^{2}\right),
$$


so that

$$
\sigma=1-\frac{1}{2}\left(h_{+}^{2}+h_{\times}^{2}\right),
$$

for $\left|h_{+}\right| \ll 1$ and $\left|h_{\times}\right| \ll 1$. The Ansatz (5b) now gives $\rho_{V}(\sigma) \propto\left(h_{+}^{2}+h_{\times}^{2}\right)^{2}$, which does not affect the standard result (8).

\subsection{Vacuum-matter energy exchange from a local action}

For homogeneous matter fields in a cosmological context, it can be shown that the vanishing covariant divergence of Eq. (4a) gives $\partial_{t} \rho_{V}=0$. A nonconstant $\rho_{V}$ apparently requires energy exchange between the vacuum energy density and the matter energy density, but this energy exchange is not present in the basic theory (11). The simplest possible way to implement this energy exchange is to change the matter action, so that the matter component by itself does not conserve energymomentum.

Consider, then, a real scalar field $\phi(x)$ with a non-dynamical dimensionless real scalar field $\zeta(x)$ in the mass-square term $(\hbar=c=1)$ :

$$
\bar{S}^{M}=-\int_{\mathbb{R}^{4}} d^{4} x \sqrt{-g}\left(\frac{1}{2} g^{\alpha \beta} \partial_{\alpha} \phi \partial_{\beta} \phi+\frac{1}{2}\left[M^{2}+m^{2} \zeta\right] \phi^{2}\right),
$$

in terms of the mass-scale $m$ of the vacuum energy density (5). The corresponding energy-momentum tensor is obtained from

$$
\bar{T}_{M}^{\alpha \beta} \equiv \frac{2}{\sqrt{-g}} \frac{\delta \bar{S}_{M}}{\delta g_{\alpha \beta}} .
$$

Using the field equation of $\phi$, the energy-momentum tensor (13) turns out to have a nonvanishing covariant divergence for nonconstant $\zeta(x)$,

$$
\nabla_{\alpha} \bar{T}_{M}^{\alpha \beta}=-\frac{1}{2} m^{2} \phi^{2} \partial^{\beta} \zeta
$$

The reason for this nonconservation is that there is no field equation for $\zeta(x)$; see, for example, the discussion around Eq. (E.1.27) in Ref. 15 where the generic matter field $\psi$ is replaced by our scalar fields $\phi(x)$ and $\zeta(x)$. Still, there is total energymomentum conservation,

$$
\nabla_{\alpha}\left(T_{V}^{\alpha \beta}+\bar{T}_{M}^{\alpha \beta}\right)=0
$$

which follows from the contracted Bianchi identities $13[15$ and the Einstein gravitational field equation (2) with $T_{V}^{\alpha \beta}$ from (4) and $\bar{T}_{M}^{\alpha \beta}$ from (13). In fact, $\zeta(x)$ is determined by (15), as shown by the explicit example in Sec. 3.2. Observe that Eq. (14) is time-reversal invariant and corresponds to a nondissipative process, different from the one considered in Ref. 10.

The modified energy-momentum conservation of the matter (14) can be expected to affect the generation of gravitational waves $\frac{16}{16}$ but this topic lies outside the scope of the present paper. Possible experimental consequences of the modified energymomentum conservation are, however, briefly discussed in Sec. 4.1 . 


\section{Cosmology}

\subsection{Metric}

Take the particular generalization of unimodular gravity given by Eqs. (1D), (5), and (12). Consider, now, the spatially-flat $(k=0)$ Robertson-Walker (RW) metric for standard comoving Cartesian coordinates and for rescaled spatial coordinates with the line element given by

$$
\begin{aligned}
d s^{2} & =-d t^{2}+a^{2}(t)\left[\left(d x^{1}\right)^{2}+\left(d x^{2}\right)^{2}+\left(d x^{3}\right)^{2}\right], \\
& =-d t^{2}+b^{2}(t)\left[\left(d \widetilde{x}^{1}\right)^{2}+\left(d \widetilde{x}^{2}\right)^{2}+\left(d \widetilde{x}^{3}\right)^{2}\right], \\
b(t) & =\frac{a(t)}{a(t)+1},
\end{aligned}
$$

where the bounded expansion factor $b(t)$ will be used from now on. Minkowski spacetime in standard Cartesian coordinates has the metric (16b) with

$$
b(t)=1 .
$$

Further rescaling of the spatial coordinates in Minkowski spacetime can, of course, give any positive constant value for $b$.

\subsection{Nonstatic universe from vacuum-matter energy exchange}

For the Robertson-Walker metric (16b) with homogeneous matter fields $\phi(t)$ and $\zeta(t)$, we get from (11), (5), and (12) the Klein-Gordon equation and the two Friedmann equations:

$$
\begin{aligned}
\partial_{t}^{2} \phi+3 H \partial_{t} \phi+M^{2} \phi & =-m^{2} \zeta \phi \\
3 H^{2} & =8 \pi G_{N}\left(\rho_{V}+\rho_{M}\right), \\
2 \partial_{t} H & =-8 \pi G_{N}\left(\rho_{M}+P_{M}\right),
\end{aligned}
$$

with the Hubble parameter, the matter energy density, the matter pressure, and the vacuum energy density given by

$$
\begin{aligned}
H & =\frac{\partial_{t} b}{b(1-b)}, \\
\rho_{M} & =\frac{1}{2}\left(\partial_{t} \phi\right)^{2}+\frac{1}{2}\left[M^{2}+m^{2} \zeta\right] \phi^{2}, \\
P_{M} & =\frac{1}{2}\left(\partial_{t} \phi\right)^{2}-\frac{1}{2}\left[M^{2}+m^{2} \zeta\right] \phi^{2}, \\
\rho_{V} & =-P_{V}=m^{4}\left[1-b^{3}\right]^{2} .
\end{aligned}
$$

Turning to the issue of vacuum-matter energy exchange, start with the time derivative of the first-Friedmann Eq. (18b),

$$
6 H \partial_{t} H=8 \pi G_{N}\left(\partial_{t} \rho_{V}+\partial_{t} \rho_{M}\right) .
$$


The left-hand-side of (19) can be eliminated by use of $3 H$ times (18c) to give the expression of total energy-momentum conservation,

$$
\partial_{t} \rho_{M}+3 H\left(\rho_{M}+P_{M}\right)=-\partial_{t} \rho_{V} .
$$

Now, the left-hand-side of (20) can be evaluated explicitly by use of the definitions (18e) and (18f) and the Klein-Gordon equation (18a). The result is

$$
\partial_{t} \rho_{M}+3 H\left(\rho_{M}+P_{M}\right)=\frac{1}{2} m^{2} \phi^{2} \partial_{t} \zeta,
$$

which, together with (20), gives

$$
\partial_{t} \rho_{V}=-\frac{1}{2} m^{2} \phi^{2} \partial_{t} \zeta .
$$

Result (21a) also follows directly from (14).

For constant $\zeta$, i.e. without essential modification of the mass-square term in the scalar action (12), the result (21b) shows that $\partial_{t} \rho_{V}$ vanishes and from (18g) we then have a static universe with $b(t)=$ const. For $\phi^{2} \partial_{t} \zeta \neq 0$, the source terms on the right-hand-sides of (21a) and (21b) show the nonvanishing energy exchange and allow for $\partial_{t} \rho_{V} \neq 0$. Equations (18g) and (21b) then imply that $\phi$ must be of order $m$, as long as $\zeta$ is of order unity.

\subsection{Numerical results}

The mass-scale $m$ of the vacuum energy density and the Planck energy $E_{P} \equiv$ $G_{N}^{-1 / 2} \approx 1.22 \times 10^{19} \mathrm{GeV}$ give the following mass-square ratio:

$$
\xi \equiv\left(m / E_{P}\right)^{2} .
$$

Next, use $E_{P}$ to define the dimensionless time $\tau$, the dimensionless Hubble parameter $h(\tau)$, the dimensionless scalar field $\varphi(\tau)$, and the dimensionless vacuum energy density $r_{V}(\tau)=\xi^{2}\left[b(\tau)^{3}-1\right]^{2}$. Henceforth, an overdot will denote differentiation with respect to $\tau$. For an initial study, we take all masses equal to the Planck scale, $M=m=E_{P}$.

Numerical results are obtained by solving the Klein-Gordon Eq. 18a), the second-Friedmann Eq. (18c), and the time derivative of the first-Friedmann Eq. (18b). This last equation, given as Eq. (19), contains the derivatives $\ddot{b}, \dot{b}, \ddot{\varphi}, \dot{\varphi}$, and, in particular, $\dot{\zeta}$. The boundary conditions at $\tau=0$ are $\{b(0), \phi(0), \dot{\phi}(0), \zeta(0)\}$ with $\dot{b}(0)$ determined by the first-Friedmann Eq. (18b). The numerical results of Fig. 1 show that Minkowski spacetime is approached with $b \rightarrow 1, h \rightarrow 0, \phi \rightarrow 0$, and $\zeta \rightarrow$ const.

Essentially the same results as in Fig. 1 are obtained for the following initial conditions:

$$
\{b(0), \phi(0), \dot{\phi}(0), \zeta(0)\}=\{1 / 2 \pm 1 / 10,3 / 2 \pm 1 / 5, \pm 1 / 100,1 \pm 1 / 100\},
$$

with corresponding $\dot{b}(0)$ values from the first-Friedmann Eq. (18b). This establishes numerically the Minkowski attractor behavior for a finite domain of initial conditions. 


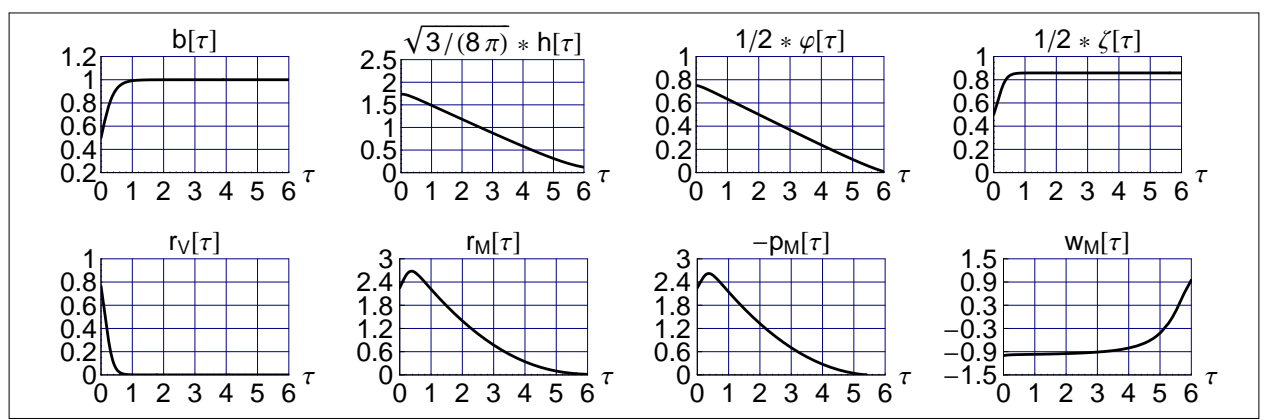

Fig. 1. Numerical solutions $b(\tau), \varphi(\tau)$, and $\zeta(\tau)$ from the ODEs (18a), (18c), and (19), with further definitions (18d), 18d, (18f), and 18g. Also shown are the following derived quantities: the Hubble parameter $h$, the vacuum energy density $r_{V}$, the matter energy density $r_{M}$, the matter pressure $p_{M}$, and the matter equation-of-state parameter $w_{M} \equiv p_{M} / r_{M}$. All masses have been taken equal to the Planck mass and the corresponding model parameter is $\xi=1$. The boundary conditions at $\tau=0$ are $\{b(0), \dot{b}(0), \phi(0), \dot{\phi}(0), \zeta(0)\}=\{1 / 2,1.25657,3 / 2,0,1\}$. The vacuum energy density $r_{V}(\tau)=\xi^{2}\left[1-b(\tau)^{3}\right]^{2}$ takes the values $r_{V}(0) \approx 0.77$ and $r_{V}(6) \approx 3 \times 10^{-13}$.

For model parameter $\xi<1$, the cosmic time unit of Fig. 1 is scaled up with a factor $1 / \xi$, which takes the approximate numerical value $1 / \xi \sim 10^{62}$ for $m \sim \mathrm{meV}$. As the time unit in Fig. 1 is the Planck time $t_{P}$, the rescaled time unit becomes $t_{P} / \xi \sim 10^{19} \mathrm{~s}$, which is relatively close (no surprise) to the inferred age of the present universe $13 \times 10^{9} \mathrm{yr} \sim 4 \times 10^{17} \mathrm{~s}$. Of course, the aim of the present work is not to give an accurate description of the actual (accelerating) universe but is more modest, namely, to investigate the gravitating vacuum energy without the introduction of new fields. Still, we have performed an exploratory calculation for a prior metric corresponding to de-Sitter spacetime; see Appendix A for details.

The model based on Eqs. (10), (5) and (12) suffices as an effective gravity theory. The ultimate question is, of course, the microscopic origin of the non-dynamical field $\zeta(x)$. Some remarks are presented in Sec. 4.2

\section{Discussion}

\subsection{Experiments}

The focus of this paper has been on cosmology, but there may also be implications for small-scale experiments. Energy-momentum conservation of any localized material system would be violated in the following way:

$$
\nabla_{\alpha} T_{M}^{\alpha \beta}=-\nabla_{\alpha} T_{V}^{\alpha \beta} \stackrel{?}{=}-\partial^{\beta} \rho_{V}\left(\sqrt{-g} / \sqrt{-g_{\text {Mink }}}\right) \stackrel{? ?}{=}-m^{4} \partial^{\beta}\left[\sqrt{-g} / \sqrt{-g_{\text {Mink }}}-1\right]^{2},
$$

with the definition $g \equiv \operatorname{det} g_{\alpha \beta}$ and the suffix "Mink" standing for the prior metric of Minkowski spacetime. The equality with a single question mark in (24) assumes 
the validity of our effective theory (11) with energy exchange from (12) and the equality with two question marks follows from the $\rho_{V}$ Ansatz (5b).

Let us get some orders of magnitude for two hypothetical experiments. We know that, at a large distance $R$ from a localized mass $M$, the order of magnitude for $\sqrt{-g} / \sqrt{-g_{\text {Mink }}}-1$ is $O\left(G_{N} M / R\right)$, and we are primarily interested in spatial derivatives of the Newtonian potential. This gives the following order of magnitude for the right-hand-side of (24):

$$
O\left(m^{4} \partial^{\beta}\left[\sqrt{-g} / \sqrt{-g_{\text {Mink }}}-1\right]^{2}\right)=O\left(m^{4} R^{-1}\left[G_{N} M / R\right]^{2}\right) .
$$

For a laboratory experiment on Earth, the Newtonian potential is $\left|\phi_{N}\right|=$ $G_{N} M / R \sim 10^{-9}$ at $R \sim 10^{7} \mathrm{~m}$. Consider, now, a quark-gluon-plasma (QGP) 17 possibly created by heavy-ion collisions at CERN's Large Hadron Collider (ALICE detector) with $\rho_{M} \sim \mathrm{GeV}^{4}$ and $L_{M} \sim \mathrm{fm}=10^{-15} \mathrm{~m}$. From (24) and (25), the relative matter energy-momentum violation (rMEMV) then has the following order of magnitude:

$$
\begin{aligned}
& \left.\operatorname{rMEMV}\right|^{(\mathrm{QGP})} \sim \frac{m^{4}}{\rho_{M}} \frac{L_{M}}{R}\left(\phi_{N}\right)^{2} \\
& \sim 10^{-88}\left(\frac{m^{4}}{10^{-12} \mathrm{eV}^{4}}\right)\left(\frac{10^{36} \mathrm{eV}^{4}}{\rho_{M}}\right)\left(\frac{L_{M}}{10^{-15} \mathrm{~m}}\right)\left(\frac{10^{7} \mathrm{~m}}{R}\right)\left(\frac{\phi_{N}}{10^{-9}}\right)^{2},
\end{aligned}
$$

for a vacuum-energy mass scale $m \sim \mathrm{meV}$ as indicated by the present accelerating universe 12

A different "experiment" concerns the binary black-hole merger (BBHM) observed by LIGO 18 Here, the metric perturbations are of order 1 and the length scale involved is of the order of the Schwarzschild radius of the smaller initial black hole, $R \sim 2 G_{N} M_{B H} \sim 60 G_{N} M_{\text {Sun }} \sim 45 \mathrm{~km}$. The effective matter energy density is of order $\rho_{M} \sim M_{B H} /\left(G_{N} M_{B H}\right)^{3} \sim E_{P}^{6} /\left(M_{B H}\right)^{2} \sim 10^{33} \mathrm{eV}^{4}$ for $M_{B H} \sim 30 M_{\text {Sun }}$. All in all, we have the following order of magnitude for the relative matter energymomentum violation:

$$
\left.\operatorname{rMEMV}\right|^{(\mathrm{BBHM})} \sim \frac{m^{4}}{\rho_{M}} \sim 10^{-45}\left(\frac{m^{4}}{10^{-12} \mathrm{eV}^{4}}\right)\left(\frac{10^{33} \mathrm{eV}^{4}}{\rho_{M}}\right) .
$$

A similar result is obtained for the (not yet observed) coalescence of two neutron stars. This neutron-star result is perhaps more reliable than the black-hole result (27a), because the matter energy density of the neutron stars can be identified directly. The result for a binary neutron-star merger (BNSM) is then

$$
\begin{aligned}
\left.\operatorname{rMEMV}\right|^{(\mathrm{BNSM})} & \sim \frac{m^{4}}{\rho_{M}}\left(\phi_{N}\right)^{2} \\
& \sim 10^{-47}\left(\frac{m^{4}}{10^{-12} \mathrm{eV}^{4}}\right)\left(\frac{10^{33} \mathrm{eV}^{4}}{\rho_{M}}\right)\left(\frac{\phi_{N}}{10^{-1}}\right)^{2} .
\end{aligned}
$$

Provided the vacuum-energy mass scale is small enough, $m \lesssim 10^{7} \mathrm{eV}$, the orders of magnitude (26) and (27) are extremely small. The predicted violation of 
matter energy-momentum conservation from (24) would then be negligible. Generally speaking, the matter-energy-momentum-violating effect from (24) would be largest for a low-density system in a strong gravitational background, with linear dimensions (length and time) of the system not very much smaller than those of the background.

\subsection{Possible underlying physics}

Condensed matter physics with emergent topologically-protected Weyl fermions (cf. Sec. 7.3 of Ref. 6 for a general discussion and Refs. 19, 20 for a detailed analysis in $2+1$ dimensions) demonstrates that known microscopic physics provides the following ingredients:

(i) formation of Weyl fermions obeying an effective gravity with metric $\widetilde{g}_{\alpha \beta}$,

(ii) nullification of the cosmological constant (vacuum energy density) in full equilibrium,

(iii) dynamical relaxation to the equilibrium Minkowski vacuum with or without oscillations of the order parameter,

(iv) mechanisms for the dissipative energy exchange between the coherent degrees of freedom (vacuum) and the incoherent degrees of freedom (matter),

(v) dependence of the vacuum energy on $\operatorname{det} \widetilde{g}_{\alpha \beta}$.

The dynamics of a freely suspended two-dimensional film 21 shows related effects for some of these items. As shown in Ref. 11, the theory of a two-dimensional film (2D brane) can be generalized to a (3+1)-dimensional theory (4D brane) with gravity and an effective $q$ field of mass dimension 4. For low-energy gravitational processes, this $4 \mathrm{D}$ brane theory reproduces the action (1) with the prior metric corresponding to a constant number density on the brane. The brane-type $q$-theory with energy scale $E_{\mathrm{br}}$ has a quadratic vacuum energy density $\rho_{V}(q)$ near equilibrium, which, in terms of the variable $\sigma$ from (1c) with $\sigma \propto 1 / q$, gives the expression $\rho_{V}(\sigma)=\left(E_{\mathrm{br}}\right)^{4} \sigma^{-2}[1-\sigma]^{2}$. The functional form of the latter expression is similar to the one of Ansatz (5b) used here.

In the present article, we have considered a generalization of unimodular gravity which naturally follows from the ingredients listed above and may come from a $(3+1)$-dimensional brane-type theory 11 The main result found here is that the vacuum-matter energy exchange is uniquely determined by the Ansatz for the vacuum energy density; see, in particular, (21b) read from right (unknown) to left (known). The reason for getting a prescribed source term of the matter equation (21a) is that there is no new field entering the vacuum energy density (18g).

But this uniquely determined vacuum-matter energy exchange is surprising from a condensed-matter-physics point of view: the energy exchange can be expected to depend on the many details of the properties of matter, and cannot be solely determined by the vacuum. Perhaps the lesson for the underlying physics of gravity is that if the microscopic degrees of freedom really give a vacuum energy density 
$\rho_{V}$ as an effective function of the macroscopic metric $g_{\alpha \beta}$ [for us, its determinant], then the microscopic degrees of freedom also arrange for the correct type of energy exchange.

\subsection{Outlook}

Without reliable information on the underlying physics of gravity, we can take a more practical point of view. The crucial input is Hubble's distance-redshift relation, interpreted as coming from an expanding universe. The present article has argued that, if gravity over large distances has a vacuum component $\rho_{V}\left(\sqrt{-g} / \sqrt{-g_{\text {Mink }}}\right)$, then a nonstatic universe requires that there exists an energy exchange between this vacuum component and the matter component. The main task is to determine (from observational cosmology or by laboratory experiments) whether or not there is a contribution $\rho_{V}\left(\sqrt{-g} / \sqrt{-g_{\text {Mink }}}\right)$ to the vacuum energy density.

\section{Acknowledgments}

It is a pleasure to thank G.E. Volovik for valuable discussions and comments.

\section{Appendix A. Prior metric of de-Sitter spacetime}

In the main text, we have used the prior metric of Minkowski spacetime. In this appendix, we take instead a prior metric corresponding to de-Sitter spacetime with a positive cosmological constant,

$$
\Lambda=m^{4} .
$$

This prior metric $g_{\alpha \beta}^{\mathrm{dS}}$ is given by (16b) with scale factor

$$
b_{\mathrm{dS}}(t)=\frac{\exp \left[\sqrt{\left(8 \pi G_{N} / 3\right) \Lambda} t\right]}{\exp \left[\sqrt{\left(8 \pi G_{N} / 3\right) \Lambda} t\right]-1+1 / b_{\mathrm{dS} 0}},
$$

where the normalization parameter $b_{\mathrm{dS} 0}=b_{\mathrm{dS} 0}(\Lambda)$ has $b_{\mathrm{dS} 0}(0)=1$ and $b_{\mathrm{dS} 0}(\Lambda)<1$ for $\Lambda>0$.

In the Lagrange density of (10) we have the potential term $\epsilon(\sigma)$, for which we take the following Ansatz

$$
\begin{aligned}
\epsilon(\sigma) & =m^{4}\left[\frac{1}{3}\left(\sigma-\frac{3}{2}\right)^{2}+\frac{5}{4}\right], \\
\rho_{V}(\sigma) & =m^{4}+m^{4}(1-\sigma)^{2}, \\
\sigma & =\sqrt{-g} / \sqrt{-g_{\mathrm{dS}}} .
\end{aligned}
$$

The matter action is still given by (12), in order to have vacuum-matter energy exchange. 


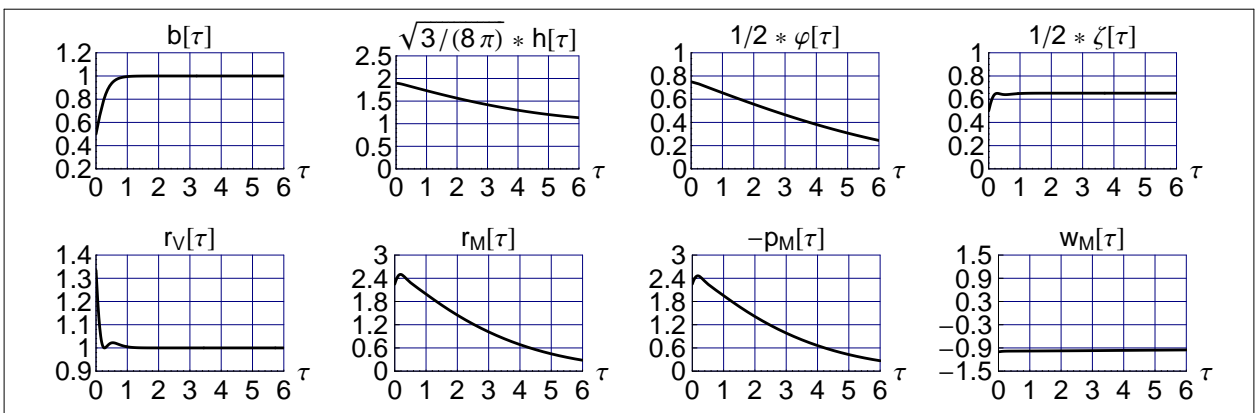

Fig. 2. Numerical solutions $b(\tau), \varphi(\tau)$, and $\zeta(\tau)$ from the ODEs (18a), 18c), and 19), with further definitions (18d), 18d, 18f and (A.4b), which includes the cosmological constant $\Lambda=m^{4}$. Also shown are the following derived quantities: the Hubble parameter $h$, the vacuum energy density $r_{V}$, the matter energy density $r_{M}$, the matter pressure $p_{M}$, and the matter equationof-state parameter $w_{M} \equiv p_{M} / r_{M}$. All masses have been taken equal to the Planck mass and the model parameters are $\xi=1$ and $b_{\mathrm{dS} 0}=2 / 3$. The boundary conditions at $\tau=0$ are $\{b(0), \dot{b}(0), \phi(0), \dot{\phi}(0), \zeta(0)\}=\{1 / 2,1.36993,3 / 2,0,1\}$. The vacuum energy density $r_{V}(\tau)$ takes the values $r_{V}(0) \approx 1.33$ and $r_{V}(6) \approx 1$.

Turning to cosmology, we take the metric (16b) and consider homogeneous matter fields $\phi(t)$ and $\zeta(t)$. The dynamic equations are again given by (18a), (18b), and (18c), with definitions (18d), (18d), and (18f). In addition, there is now the following vacuum energy density with cosmological constant (A.1) included, first in terms of dimensional variables and then in terms of dimensionless variables:

$$
\begin{aligned}
& \rho_{V}(t)=-P_{V}(t)=m^{4}\left[1+\left(1-b(t)^{3} / b_{\mathrm{dS}}(t)^{3}\right)^{2}\right], \\
& r_{V}(\tau)=-p_{V}(\tau)=\xi^{2}\left[1+\left(1-b(\tau)^{3} / b_{\mathrm{dS}}(\tau)^{3}\right)^{2}\right] .
\end{aligned}
$$

Numerical results are shown in Fig. 2, where the Hubble parameter $H(t)$ approaches the constant value $\sqrt{\left(8 \pi G_{N} / 3\right) \Lambda}$ and the matter field $\phi(t)$ approaches 0 while keeping its equation-of-state parameter $w_{M}$ close to -1 .

\section{References}

1. J.J. van der Bij, H. van Dam, and Y.J. Ng, "The exchange of massless spin two particles," Physica 116A (1982) 307.

2. A. Zee, "Remarks on the cosmological constant problem," in: S.L. Mintz and A. Perlmutter (eds.), High-Energy Physics: Proceedings of Orbis Scientiae 1983 (Plenum Press, N.Y., 1985), p. 211.

3. W. Buchmüller and N. Dragon, "Einstein gravity from restricted coordinate invariance," Phys. Lett. B 207 (1988) 292.

4. M. Henneaux and C. Teitelboim, "The cosmological constant and general covariance," Phys. Lett. B 222 (1989) 195.

5. S. Weinberg, "The cosmological constant problem," Rev. Mod. Phys. 61 (1989) 1, Sec. VII. 
6. G.E. Volovik, The Universe in a Helium Droplet, Paperback Edition (Oxford University Press, 2008).

7. F.R. Klinkhamer and G.E. Volovik, "Self-tuning vacuum variable and cosmological constant," Phys. Rev. D 77 (2008) 085015, arXiv:0711.3170.

8. F.R. Klinkhamer and G.E. Volovik, "Dynamic vacuum variable and equilibrium approach in cosmology," Phys. Rev. D 78 (2008) 063528, arXiv:0806.2805.

9. F.R. Klinkhamer and G.E. Volovik, "Towards a solution of the cosmological constant problem," JETP Lett. 91 (2010) 259, arXiv:0907.4887.

10. F.R. Klinkhamer and G.E. Volovik, "Dynamic cancellation of a cosmological constant and approach to the Minkowski vacuum," to appear in Mod. Phys. Lett. A, arXiv:1601.00601.

11. F.R. Klinkhamer and G.E. Volovik, "Brane realization of $q$-theory and the cosmological constant problem," JETP Lett. 103 (2016) 627, arXiv:1604.06060.

12. K.A. Olive et al. [Particle Data Group Collaboration], "Review of particle physics," Chin. Phys. C 38 (2014) 090001, Sec. 26.

13. C.W. Misner, K.S. Thorne, and J.A. Wheeler, Gravitation (W.H. Freeman and Co., New York, 1973).

14. C.M. Will, Theory and Experiment in Gravitational Physics, Revised Edition (Cambridge University Press, Cambridge, UK, 1993), Sec. 5.5.

15. R.M. Wald, General Relativity (Chicago University Press, Chicago, 1984).

16. E. Poisson and C.M. Will, Gravity: Newtonian, Post-Newtonian, Relativistic (Cambridge University Press, Cambridge, UK, 2014), Sec. 11.2.1.

17. L.D. McLerran, "The physics of the quark-gluon plasma," Rev. Mod. Phys. 58 (1986) 1021.

18. B.P. Abbott et al. [LIGO Scientific Collaboration and Virgo Collaboration], "Observation of gravitational waves from a binary black hole merger," Phys. Rev. Lett. 116 (2016) 061102, arXiv:1602.03837

19. M.S. Foster, M. Dzero, V. Gurarie, and E.A. Yuzbashyan, "Quantum quench in a p+ip superfluid: Winding numbers and topological states far from equilibrium," Phys. Rev. $B 88$ (2013) 104511, arXiv:1307.1485.

20. M.S. Foster, V. Gurarie, M. Dzero, and E.A. Yuzbashyan, "Quench-induced Floquet topological p-wave superfluids," Phys. Rev. Lett. 113 (2014) 076403, arXiv:1307.2256.

21. E.I. Kats and V.V. Lebedev, "Nonlinear fluctuation effects in dynamics of freely suspended films," Phys. Rev. E 91 (2015) 032415, arXiv:1501.06703. 